\title{
Higher-order perturbation theory for the diffusion equation in heterogeneous media: application to layered and slab geometries
}

\author{
Angelo Sassaroli, ${ }^{1, *}$ Fabrizio Martelli, ${ }^{2}$ and Sergio Fantini ${ }^{1}$ \\ 'Department of Biomedical Engineering, Tufts University, 4 Colby Street, Medford, Massachusetts, USA \\ ${ }^{2}$ Dipartimento di Fisica dell'Universitá degli Studi di Firenze, Via G. Sansone 1, 50019 Sesto Fiorentino, Firenze, Italy \\ ${ }^{*}$ Corresponding author: angelo.sassaroli@tufts.edu
}

Received 2 September 2008; revised 21 November 2008; accepted 29 November 2008; posted 2 December 2008 (Doc. ID 100786); published 15 January 2009

\begin{abstract}
We apply a previously proposed perturbation theory of the diffusion equation for studying light propagation through heterogeneous media in the presence of absorbing defects. The theory is based on the knowledge of (a) the geometric characteristics of a focal inclusion, (b) the mean optical path length inside the inclusion, and (c) the optical properties of the inclusion. The potential of this method is shown in the layered and slab geometries, where calculations are carried out up to the fourth order. The relative changes of intensity with respect to the unperturbed (heterogeneous) medium are predicted by the theory to within $10 \%$ for a wide range of contrasts $d \Delta \mu_{a}$ (up to $d \Delta \mu_{a} \approx 0.4-0.8$ ), where $d$ is the effective diameter of the defect and $\Delta \mu_{a}$ the absorption contrast between defect and local background. We also show how the method of Padé approximants can be used to extend the validity of the theory for a larger range of absorption contrasts. Finally, we study the possibility of using the proposed method for calculating the effect of a colocalized scattering and absorbing perturbation. () 2009 Optical Society of America

OCIS codes: $\quad 170.3660,170.5280,170.7050$.
\end{abstract}

\section{Introduction}

Near-infrared spectroscopy (NIRS) and diffuse optical tomography (DOT) are optical techniques that are currently the subjects of intensive research because of the unique characteristics of near-infrared light when it is used to probe biological tissues. Nearinfrared light is noninvasive, it can penetrate rather deeply in tissues $(\approx 2 \mathrm{~cm})$, and it is highly sensitive to changes in the concentration of oxyhemoglobin and deoxyhemoglobin. NIRS also features a high temporal resolution (a few tenths of a millisecond), which allows the detailed monitoring of relevant physiological parameters. However, the propagation of nearinfrared light in biological tissues is governed mainly by the phenomenon of scattering; therefore the image quality of internal structures is naturally

0003-6935/09/100D62-12\$15.00/0

(C) 2009 Optical Society of America deteriorated, and the spatial resolution is no better than about $5 \mathrm{~mm}$.

The main challenge that NIRS and DOT are facing is the accurate mapping of the optical properties (namely, the absorption and reduced scattering coefficients) from a series of measurements taken at the boundary of a target organ. This inverse problem is fundamentally ill posed [1]. Most of the solutions to the inverse problem in DOT rely on accurate and possibly fast forward models of photon migration in tissues. Owing to the diffusive nature of light propagation in tissues, the diffusion equation (DE) is widely used to predict measurements of data types at different detector's sites. Typical data types are the intensity in the continuous wave (CW) domain, amplitude and phase lag in the frequency domain, and the distribution of time of flight of detected photons in the time domain. Analytical solutions of the $\mathrm{DE}$ are found for homogeneous media having regular geometries, such as the infinite, semi-infinite, 
and slab [2]. All of these geometries are currently used for describing light propagation in several tissue organs (e.g., breast, head, forearm, and calf) whenever we want to obtain information on the average bulk concentration of oxyhemoglobin and deoxyhemoglobin and tissue oxygen saturation. The DE has also been solved analytically for several regularly bounded homogeneous media including single regularly shaped defects [3-ㅡㄴ media [7-10]. These solutions are used for describing the effect of tumors in the breast and for describing light propagation in the head, respectively. For imaging small tissue volumes or tissues containing clear (low absorbing and low scattering) regions, the radiative transfer equation (RTE) has also been used [11].

More realistic tissue geometries require numerical methods for solving the DE or RTE. Among these methods are the finite-element method [12-14], the finite-difference method [15], and the boundary element method [9]. These methods, despite their flexibility in dealing with complex geometries, are rather time consuming, especially for 3D calculations. Another effective method to study light transport in tissues which is also capable of dealing with complex geometries is the Monte Carlo method (MC) [16-19]. The MC method has the drawback of being rather time consuming, and it is not usually implemented as a forward model for image reconstruction. However, the MC method is simple and reliable, and for these reasons it has been used largely for assessing the validity of other more complex numerical methods.

First and $N$ th-order perturbation theories for the DE have also been proposed for studying photon migration in tissues [20-25]. In some early work [20,21] the authors introduced the idea of improving the results of perturbation theory by using iterations of first-order theory to correct the fluence rate and the output flux. In the same studies calculations of light propagation in the presence of single absorbing or scattering defects were carried out in the frequency domain for the infinite medium geometry. In more recent work $[24,25]$ higher-order perturbation theory has been studied in the time domain for the slab geometry including single absorbing and/or scattering defects, and simple inversion procedures have been proposed to study the optical properties of breast tumors. Most of the approaches to higher-order perturbation theory proposed in the literature adopted the strategy of repeated iterations of first-order calculations (the Born approximation). This method implies the calculation of multiple integrals of the same order of the perturbation theory addressed. For example a second-order theory requires the calculation of a double integral in the volume of the defect, a thirdorder theory requires the calculation of a triple integral in the volume of the defect, etc. In contrast our approach to perturbation theory has two main points: (a) we showed that rearranging those multiple integrals can yield the expression for higherorder path-length moments, which offer a more intuitive viewpoint for studying absorbing perturbations [22]; (b) we proposed a heuristic formula to reduce the calculation of multiple integrals to a single one. We mention that other groups also used heuristic methods based on some correction factors to improve the results of the Born approximation $[26,27]$.

In our previous work [22] we proposed a method to describe the output flux in presence of single or multiple absorbing defects embedded in a background medium having an arbitrary geometry. The method is based on the calculation of the moments of the generalized temporal point spread function, which requires knowledge of the Green's function for the fluence rate and output flux. Explicit formulas were given in the three domains of investigation, and numerical results in the presence of single and multiple absorbing defects were shown in the $\mathrm{CW}$ domain and semi-infinite geometry. The key point of the proposed theory is an approximate formula for calculating the self-moments (i.e., the moments $\left\langle l_{i}^{n}\right\rangle n>1$ as $\left\langle l_{i}^{2}\right\rangle,\left\langle l_{i}^{3}\right\rangle$, etc., where $l_{i}$ is a random variable associated with the path length traveled inside a defect by a detected photon. More specifically $\left\langle l_{i}^{n}\right\rangle$ is calculated by using (a) the value of the mean path length $\left\langle l_{i}\right\rangle$ inside a defect, (b) the value of the photon energy in the volume occupied by the defect when a point source is located at its center, and (c) some unitless scaling parameters.

In other work [23] we showed that the scaling parameters are rather insensitive to the shape and size of the defect (at least when $d_{\min } \approx d_{\max }$, where $d_{\min }$ and $d_{\max }$ are the minimum and maximum chord lengths connecting two points at the boundary of the defects) and of the geometrical and optical properties of the otherwise homogeneous background medium. Explicit values of the scaling parameters were calculated up to the fourth-order moment, $\left\langle l_{i}^{4}\right\rangle$. These formulas guided us to the insight that the scaling parameters are also rather robust in the presence of strong heterogeneities of the background medium and prompted us to study our method's performance in the layered and slab geometries.

In Section $\underline{2}$ we present a concise overview of the theory, and we rewrite the proposed heuristic formula for the calculation of the self-moments. Based on the physical meaning of this formula, we speculate that the theory may be used as a flexible way to describe the output flux in more complex geometries and distribution of the optical properties. In Section 3 we show some results in the CW domain for two- an $\bar{d}$ three-layered geometries and also for slab geometry characterized by a heterogeneous background. The results predicted by the theory are validated by means of MC simulations. We also show how the method of Padé approximants $[25,28]$ can be used to further extend the limitations of the proposed fourth-order perturbation theory. Finally, we investigate the possibility of using the proposed formula for studying the effect of a colocalized scattering and absorbing inclusion. In Section 4 we draw the conclusions and discuss future applications of the theory. 


\section{Theory}

In our previous work [22] we proposed a heuristic formula for the calculation of the self-moment of order $n$ inside a defect $\left(\left\langle l_{i}^{n}\right\rangle\right)$ :

$$
\left\langle l_{i}^{n}\right\rangle \approx c_{n-1}\left\langle l_{i}\right\rangle\left(\int_{V_{i}} \phi_{0}\left(\mathbf{r}, \mathbf{r}_{i}\right) \mathrm{d} \mathbf{r}\right)^{n-1}, \quad n>1,
$$

where $\phi_{0}\left(\mathbf{r}, \mathbf{r}_{i}\right)$ is the Green's function of the baseline medium for the fluence rate (normalized to the source strength, i.e., $\left[\phi_{0}\right]=L^{-2}$, where $L$ is the length) when the pointlike source is at the center of the defect $\left(\mathbf{r}_{i}\right), c_{n-1}$ are unitless scaling parameters, and $\left\langle l_{i}\right\rangle$ is the mean path length of detected photons inside the defect. We have also studied the effect on the detected intensity of a single or multiple defects embedded in otherwise homogeneous media [23]. For the calculation of the integral in Eq. (1) we applied the method of the images; however we found that for locations of the defect farther than about $5 \mathrm{~mm}$ from the external boundary, the calculation can be carried out by using the Green's function of infinite medium geometry with the optical properties of the background medium. For these locations of the defect the error induced by neglecting negative point sources was less than 1\%. By using extensive MC simulations in the slab and semi-infinite geometries for several defect's positions and sizes $(1 \mathrm{~mm} \leq r \leq$ $5 \mathrm{~mm}, r$ being an effective radius of the defect) and a wide range of optical properties of the background medium $\left(0.5 \mathrm{~mm}^{-1} \leq \mu_{s}^{\prime} \leq 2 \mathrm{~mm}^{-1}, 0.005 \mathrm{~mm}^{-1} \leq \mu_{a} \leq\right.$ $0.05 \mathrm{~mm}^{-1}$ ) we found that the variances of the scaling parameters are rather small and most of their values lie in the ranges 1.48-1.58, 3.1-3.7, and 8.5-11.5 for $c_{1}, c_{2}$, and $c_{3}$, respectively [23]. Explicit values used here are $c_{1}=1.53, c_{2}=\overline{3.4}$, and $c_{3}=10$. A brief summary of the perturbative $\mathrm{DE}$ can be found in Appendix A.

If we want to use Eq. (1) for general heterogeneous media, it would make sense to choose as the integrand the Green's function of the baseline (unperturbed) heterogeneous medium under investigation. However, an important physical insight into Eq. (1) is that while the mean path length $\left(\left\langle l_{i}\right\rangle\right)$ depends on the global distribution of the optical properties of the medium between a given sourcedetector pair, the other factor (i.e., the integral) depends mainly on the local optical properties of the defect. Therefore we hypothesize that Eq. (1) can also be used for heterogeneous background me $\bar{d}-$ ia with the same approximations described above. This is an important point because it allows a significant simplification of the calculations. Since these arguments are valid for arbitrary background media, we speculate that Eq. (1) may be used as a flexible method to extend the limits of first-order perturbation theory without adding any computational burden even for heterogeneous media. The values of the self-moments $\left\langle l_{i}^{n}\right\rangle(n=2,3,4)$ calculated with Eq. (1) were used in two ways: a. For the calculation of MacLaurin series expansion of the intensity change $\left(\Delta I / I_{0}\right)$ as a function of the absorption contrast $\Delta \mu_{a}$ between defect and local background $[\underline{22}, \underline{23}]$,

$$
\begin{aligned}
\frac{\Delta I\left(\Delta \mu_{\mathrm{a}}\right)}{I_{0}} \approx & -\left\langle l_{i}\right\rangle \Delta \mu_{a}+\frac{1}{2 !}\left\langle l_{i}^{2}\right\rangle \Delta \mu_{a}^{2}-\frac{1}{3 !}\left\langle l_{i}^{3}\right\rangle \Delta \mu_{a}^{3} \\
& +\frac{1}{4 !}\left\langle l_{i}^{4}\right\rangle \Delta \mu_{a}^{4} .
\end{aligned}
$$

In Eq. (2) $\Delta I\left(\Delta \mu_{a}\right)=I\left(\Delta \mu_{a}\right)-I_{0}$, where $I\left(\Delta \mu_{a}\right)$ and $I_{0}$ are the detected intensities when the absorption contrast is $\Delta \mu_{a}$ and 0 , respectively. Note that all the moments are calculated in the initial state of the medium: $\Delta \mu_{a}=0$.

b. For the method of Padé approximants [29]. This method uses the coefficients of MacLaurin expansion of $\Delta I / I_{0}$ to find a rational function $P_{M / N}\left(\Delta \mu_{a}\right)$, of order $M+N+1$, that extends the range of convergence of the truncated series [Eq. (2)]. The rational function is expressed as

$$
\frac{\Delta I\left(\Delta \mu_{a}\right)}{I_{0}} \approx P_{M / N}\left(\Delta \mu_{a}\right)=\frac{\sum_{k=0}^{M} a_{k} \Delta \mu_{a}^{k}}{1+\sum_{k=1}^{N} b_{k} \Delta \mu_{a}^{k}} .
$$

For our results we used $M=N=2$, because only five coefficients can be derived from Eq. (2) (the first being 0 ). The coefficients $a_{k}$ and $b_{k}$ are calculated by imposing that the value of $P_{M / N}\left(\Delta \mu_{a}\right)$, and its first $M+N$ derivatives calculated at $\Delta \mu_{a}=0$ coincide with those of $\Delta I / I_{0}$ [Eq. (2)]. The method of Padé approximants, by using the same coefficients of the MacLaurin expansion, has the ability to extend the convergence of the approximating function for a wider range of the independent variable $\Delta \mu_{a}$. It is a somewhat efficient but uncontrolled method because it is not possible to know exactly its range of convergence [29]. We note that the rational function in Eq. (3) has two poles in the complex field. When one of the poles becomes real (zero), the plot of $\Delta I / I_{0}$ against $\Delta \mu_{a}$ shows a vertical asymptote. Our study indicates that zeros are sensitive to the coefficient of MacLaurin expansion. In the discussion we will address this limitation of Padé approximants and also a possible solution.

We used MC simulations for validation of the theory. Details of the MC code were provided in previous work [23,30,31]. MC simulations were used for two purposes: (a) as a correct forward model of light propagation to calculate the values of $\Delta I / I_{0}$ for a wide range of absorption contrasts $\Delta \mu_{a}$ and (b) for the direct calculation of the self-moments [23] $\left\langle l_{i}^{n}\right\rangle(n=1$, $2,3,4)$, the first of which was used in Eq. (1) for deriving the other moments independently. For the MC results the simulated photons (those injected into the medium) were running until we detected 10,000 photons in each detector. The detected photons were used for the calculation of re-emitted intensity (I); therefore its estimated error is $1 \%$ for the baseline 
medium. The error increases up to $5 \%-10 \%$ as we consider larger absorption contrasts between defect and medium, up to $\Delta \mu_{a}=0.2-0.3 \mathrm{~mm}^{-1}$. We note that there is an alternative way to calculate $\left\langle l_{i}\right\rangle$ that uses one of the available solutions for the Green's function of the $\mathrm{DE}$ in the layered or slab geometries $[30, \underline{31}]$.

\section{Results}

In Fig. 1 is shown a schematic of a two-layer medium and two cylindrical defects that we considered for our results in the layered geometry. The thickness of the first layer is $10 \mathrm{~mm}$, while the second layer, unless otherwise specified, is infinite. The diameter and height of cylinder $A$ are $6 \mathrm{~mm}$, while the diameter and height of cylinder $\mathrm{B}$ are 11.2 and $3 \mathrm{~mm}$, respectively. We chose cylinder A because the minimum and maximum chord lengths are similar $\left(d_{\min } \approx\right.$ $\left.d_{\max }\right)$, and the scaling parameters $c_{n-1}$ found in our previous work [23] should apply for this case. Cylinder B was chosen as a model region of brain activation in neuroimaging studies. For the calculation of total photon energy inside the defect [the integral in Eq. (1)], we carried out a numeric integration over parallelepipeds having the same volumes and heights of the two cylinders. In the following results only one of the two defects is present in any one test case, and its position was changed to validate our theory. To describe the size of these cylinders with only one parameter, we chose to use an effective diameter $d$, which is defined as the diameter of a spherical defect having the same volume as the cylindrical defects.

In Fig. 2 are plotted the changes of relative intensity $\Delta I / I_{0}^{-}$as the function of the absorption contrast

\section{SCHEMATIC OF TWO-LAYER MEDIUM + DEFECTS}

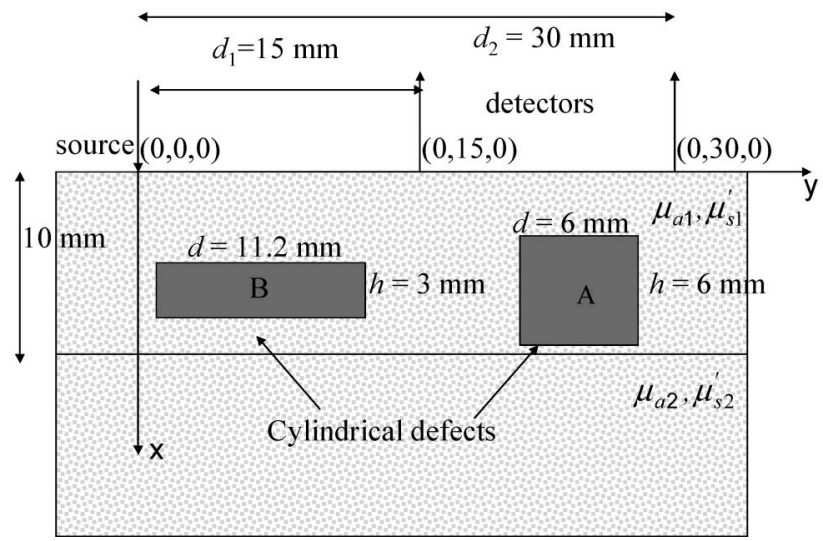

Fig. 1. Schematic of two-layer medium and cylindrical defects used for the simulations. The thickness of the first layer is $10 \mathrm{~mm}$, while the second layer is infinite. The diameter and height of cylinder A are $6 \mathrm{~mm}$, while the diameter and height of cylinder B are 11.2 and $3 \mathrm{~mm}$, respectively. The input point of the light source is the origin of the reference system, and the two detectors have coordinates $(x, y, z)=(0,15,0) \mathrm{mm}$ and $(x, y, z)=(0,30,0) \mathrm{mm}$, respectively. The absorption and reduced scattering coefficient of the first and second layers are $\mu_{a 1}, \mu_{s 1}^{\prime}$ and $\mu_{a 2}, \mu_{s 2}^{\prime}$, respectively. Only one of the two defects is present in any one test case.
$\Delta \mu_{\alpha}$ between defect and local background medium. The six plots refer to the four orders of the proposed theory, to the Padé approximants, and to MC results. The optical properties of the layers are $\mu_{a 1}=$ $0.01 \mathrm{~mm}^{-1}, \mu_{s 1}^{\prime}=0.5 \mathrm{~mm}^{-1}$, and $\mu_{a 2}=0.015 \mathrm{~mm}^{-1}$, $\mu_{s 2}^{\prime}=1 \mathrm{~mm}^{-1}$ for the first and second layer, respectively. The defect is cylinder A (Fig. 1), which is located in the top layer, with the center at $(x, y, z)=$ $(6.5,8,0) \mathrm{mm}$. We note that the lower surface of the cylinder is only $0.5 \mathrm{~mm}$ from the border between top and bottom layers. In Figs. 2(a) and 2(b) are plotted the changes of relative intensity at sourcedetector distances of 15 and $30 \mathrm{~mm}$, respectively. In Figs. 2(c) and 2(d) are plotted the same curves of Figs. 2(a) and 2(b), respectively, on expanded scales. From Figs. 2(a) and 2(b) we can see that increasing orders of the proposed perturbation theory show an increasing agreement with the MC curve: up to an absorption contrast of $\Delta \mu_{a}=0.1 \mathrm{~mm}^{-1}$, the discrepancy between the fourth-order theory and $\mathrm{MC}$ curves is less than $12 \%$, while first-order theory shows a discrepancy of up to $65 \%$. We also note that the discrepancy between MC and Padé curves is less than 1.5\%. In Figs. 2(c) and 2(d) the agreement between perturbation theory and $\mathrm{MC}$ results becomes increasingly worse for larger absorption contrasts, as should be expected. However we can see that Padé approximants show an agreement with MC results better than 3\% even for very large absorption contrasts. We stress that the good agreement found between our theoretical results and MC simulations are uniquely due to the accuracy with which the higher-order self-moments are calculated by Eq. (1). In Table 1 are written the values of the moments calculated by MC code and by the proposed theory. Within the statistical errors of the MC results we can say that the values of the self-moments calculated by the theory match those calculated by the MC code. We also repeated the simulation for a different choice of the reduced scattering coefficients (all the other parameters were fixed), $\mu_{\mathrm{s} 1}^{\prime}=$ $1.5 \mathrm{~mm}^{-1}, \mu_{s 2}^{\prime}=1 \mathrm{~mm}^{-1}$ for the first and second layer, respectively, and we found that the agreement of fourth-order theory with MC results was better than $10 \%$ for absorption contrasts up to $\Delta \mu_{a}=$ $0.045 \mathrm{~mm}^{-1}$. Padé approximants again showed the best agreement with MC results with a discrepancy better than $8 \%$ for absorption contrasts up $\Delta \mu_{a}=$ $0.22 \mathrm{~mm}^{-1}$.

In Fig. 3 we plot the changes of relative intensity $\Delta I / I_{0}$ against $\Delta \mu_{a}$ between the defect and the local background medium for the same medium of Fig. 2, when defect $\mathrm{A}$ is located in the bottom layer, with the center at $(x, y, z)=(13.5,8,0) \mathrm{mm}$. For this case the moments are slightly overestimated by the theory, and consequently the third-order calculations show a slightly better agreement with $\mathrm{MC}$ results than the fourth order's; however both orders' calculations are within $10 \%$ of the MC curve for $\Delta \mu_{a}<0.06 \mathrm{~mm}^{-1}$. If we use the self-moments calculated directly by the MC code in Eq. (2), the different orders of 

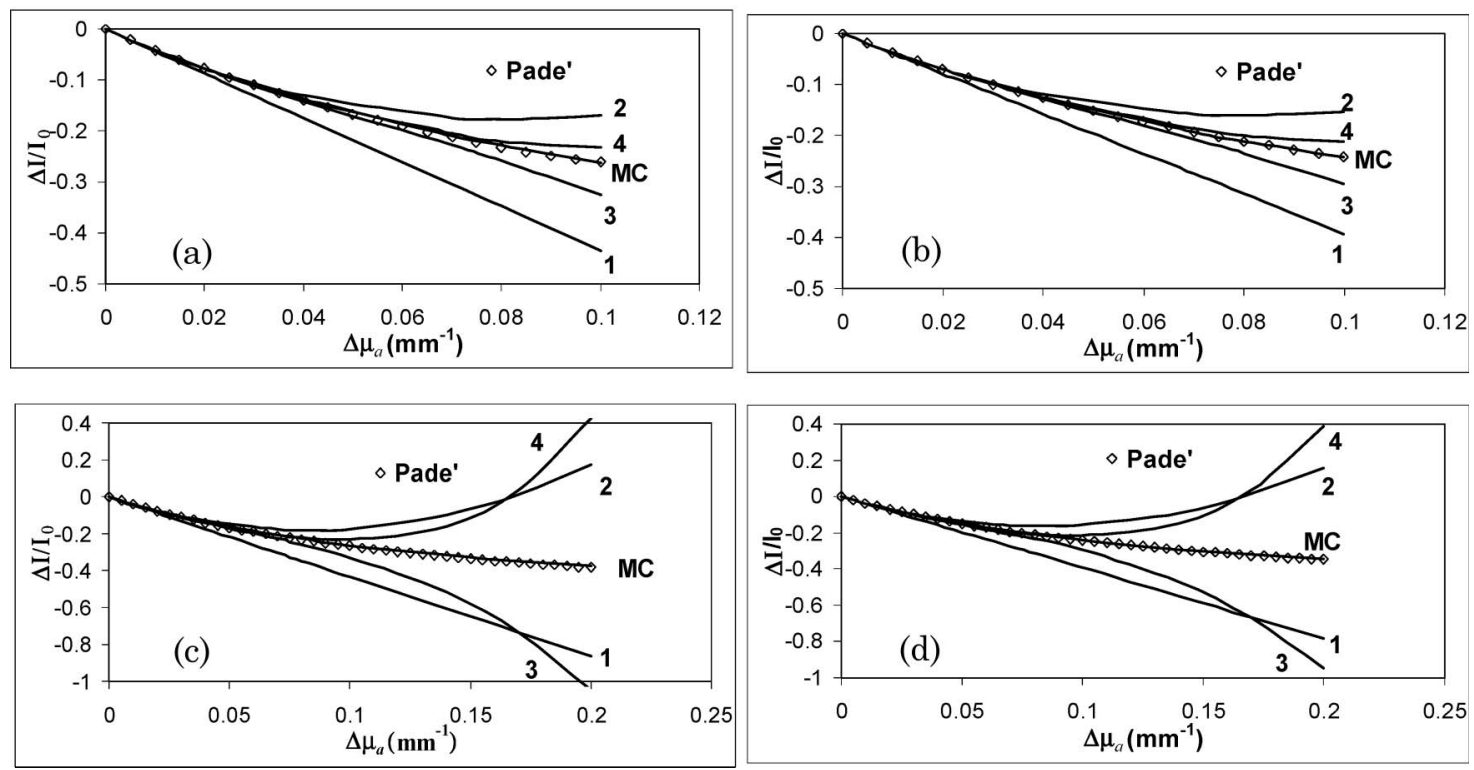

Fig. 2. The changes of relative intensity $\left(\Delta I / I_{0}\right)$, obtained with different orders of perturbation theory, with Padé approximants and with MC, are plotted against the changes of absorption contrast $\Delta \mu_{a}$ between cylinder A (Fig. 1 ) and the local background medium. The thickness of the first layer is $10 \mathrm{~mm}$, and the optical properties are $\mu_{a 1}=0.01 \mathrm{~mm}^{-1}, \mu_{s 1}^{\prime}=0.5 \mathrm{~mm}^{-1}$, and $\mu_{a 2}=0.015 \mathrm{~mm}^{-1}, \mu_{s 2}^{\prime}=1 \mathrm{~mm}^{-1}$, for the first and second layer, respectively. The defect is located in the top layer, with the center at $(x, y, z)=(6.5,8,0) \mathrm{mm}$. Shown are the changes of intensity at the (a) shortest $(15 \mathrm{~mm})$ and $(\mathrm{b})$ farthest $(30 \mathrm{~mm})$ source-detector distance. In $(\mathrm{c})$ and $(\mathrm{d})$ the same curves are plotted on an expanded scale.

perturbation theory show the expected behavior, and the discrepancy between the fourth order and the MC results is within $10 \%$ for $\Delta \mu_{a}<0.07 \mathrm{~mm}^{-1}$. A similar result is obtained by using a different choice of the scaling parameters: $c_{1}=1.47, c_{2}=3.1, c_{3}=8.5$. We note that the new parameters are still within the range found in our previous paper [23] and reported in the Section 2. Padé approximants show the best overall agreement with MC results, with a discrepancy less than $10 \%$ for a wide range of absorption contrasts up to $\Delta \mu_{a}=0.2 \mathrm{~mm}^{-1}$ as shown on the expanded scales [Figs. 3(c) and 3(d)]. Also for this position of the defect we ran another simulation with $\mu_{s 1}^{\prime}=1.5 \mathrm{~mm}^{-1}, \mu_{s 2}^{\prime}=1 \mathrm{~mm}^{-1}$ (all the other parameters were not changed) for the first and second layer, respectively. The agreement of fourth-order theory with MC results was better than $7 \%$ for absorption contrasts up to $\Delta \mu_{a}=0.055 \mathrm{~mm}^{-1}$, while Padé approximants again showed the best agreement with $\mathrm{MC}$ results with a discrepancy better than $6 \%$ for absorption contrasts up $\Delta \mu_{a}=0.2 \mathrm{~mm}^{-1}$. We note that the different quantitative agreements

Table 1. Calculated Values of the Moments ${ }^{a}$

\begin{tabular}{ccccc}
\hline & \multicolumn{2}{c}{ MC Code } & \multicolumn{2}{c}{ Proposed Theory } \\
\cline { 2 - 4 } Moment & $d_{1}=15 \mathrm{~mm}$ & $d_{2}=30 \mathrm{~mm}$ & $d_{1}=15 \mathrm{~mm} d_{2}=30 \mathrm{~mm}$ \\
\hline$\left\langle l_{1}^{2}\right\rangle\left(\mathrm{mm}^{2}\right)$ & $55 \pm 6$ & $48 \pm 5$ & 53 & 48 \\
$\left\langle l_{1}^{3}\right\rangle\left(\mathrm{mm}^{3}\right)$ & $980 \pm 150$ & $830 \pm 130$ & 941 & 854 \\
$\left\langle l_{1}^{4}\right\rangle\left(\mathrm{mm}^{4}\right)$ & $23,000 \pm 5000$ & $19,000 \pm 5000$ & 22,125 & 20,080 \\
\hline
\end{tabular}

${ }^{a}$ The medium and the defect are those of Fig. $\underline{2}$. between $n$ th-order theory and MC results are mostly intrinsic to $n$ th-order theory and depend only slightly on the approximations inherent in Eq. (1). In other words the accuracy of $n$ th-order theory, given a certain absorption contrast and volume of the defect, is a quite complex function of its location and also of the background optical properties.

In the following results we also tried to test the robustness of Eq. (1) for defects having different minimum and maximum chord lengths $\left(d_{\min } \ll d_{\max }\right)$, such as cylinder B of Fig. 1. In Fig. $\underline{4} \Delta I / I_{0}$ is plotted against the absorption contrast $\overline{\Delta \mu}$ for the background medium of Fig. 2 when cylinder B is placed in the top layer, with the center at $(x, y, z)=$ $(7.5,8,0) \mathrm{mm}$. Fourth-order theory and Padé approximants show an agreement with MC results better than $10 \%$ and $11 \%$, respectively, at both sourcedetector distances up to an absorption contrast of $\Delta \mu_{a}=0.095 \mathrm{~mm}^{-1}$. However, a closer look at the fourth-order plots of Figs. 4(a) and 4(b) also reveals that some moments are slightly underestimated by the theory, since the fourth-order and MC curves intersect each other. Padé approximants show an agreement with MC results better than $10 \%$ for $\Delta \mu_{a} \leq 0.095 \mathrm{~mm}^{-1}$ and better than $20 \%$ up to an absorption contrast of $\Delta \mu_{a}=0.29 \mathrm{~mm}^{-1}$.

In Fig. 5 , for the same medium and defect of Fig. 4 , $\Delta I / I_{0}$ is plotted against the absorption contrast $\Delta \bar{\mu}_{a}$ when the defect is located in the bottom layer, with the center at $(x, y, z)=(12.5,8,0) \mathrm{mm}$. Fourth-order calculations have an agreement with $\mathrm{MC}$ results better than $10 \%$ for $\Delta \mu_{a} \leq 0.055 \mathrm{~mm}^{-1}$. In the same 
$d_{1}$
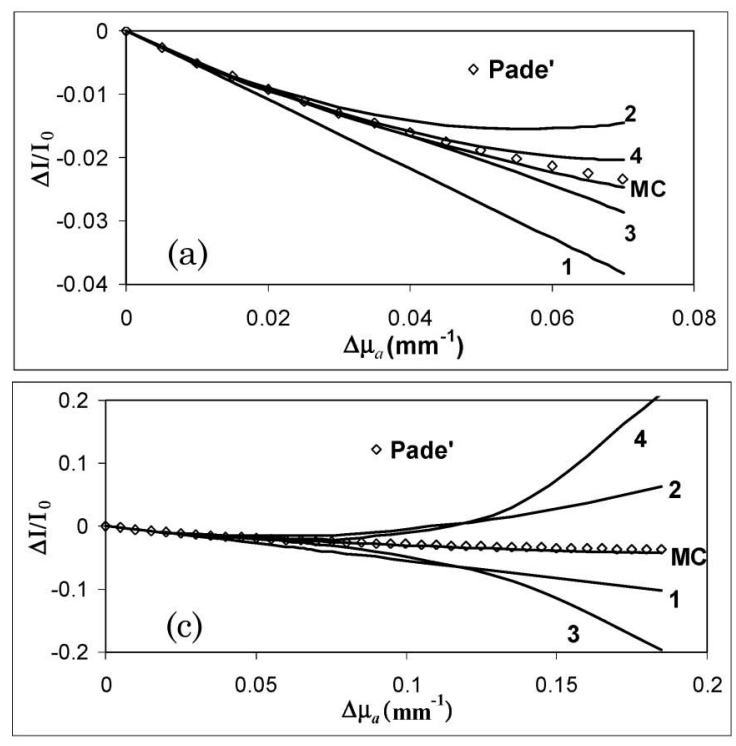

$d_{2}$
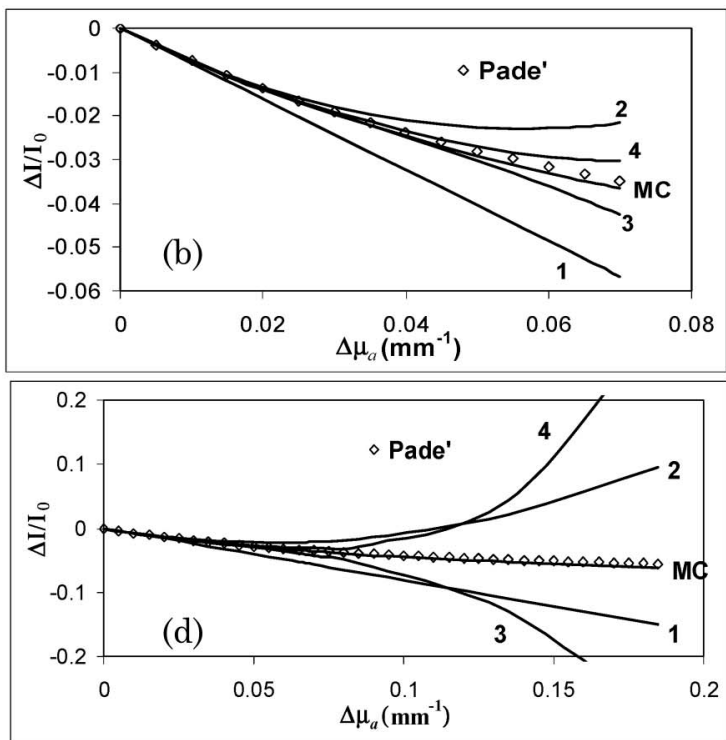

Fig. 3. As in Fig. $\underline{2}$, but the defect is located in the bottom layer, with the center at $(x, y, z)=(13.5,8,0) \mathrm{mm}$.

range Padé approximants have an agreement with MC results better than $5 \%$ and $0.2 \%$ at the source-detector distance of 15 and $30 \mathrm{~mm}$, respectively. For larger absorption contrast perturbation theory has a wider discrepancy with MC results, while Padé approximants show the best results: the agreement with $\mathrm{MC}$ curves is better than $10 \%$ and $3 \%$ at the shortest and longest source-detector distances, respectively, for $\Delta \mu_{a} \leq 0.23 \mathrm{~mm}^{-1}$.

In Fig. 6 we carried out the calculations for a threelayer medium that can be used as another simplified model of the head. The thickness of the first layer, where scalp and skull are lumped together, is $8 \mathrm{~mm}$, while the thin middle layer having thickness of $2 \mathrm{~mm}$ represents the cerebrospinal fluid. The bottom layer, $(20 \mathrm{~cm}$ thick) for the purpose of our calculation can be considered infinitely extended, and it represents the brain where the defect (cylinder B) simulates a focal hemodynamic change. The optical properties of the layers are $\Delta \mu_{a}=0.01 \mathrm{~mm}^{-1}$, $\mu_{s 1}^{\prime}=0.5 \mathrm{~mm}^{-1}, \mu_{a 2}=0.002 \mathrm{~mm}^{-1}, \mu_{s 2}^{\prime}=0.1 \mathrm{~mm}^{-1}$, $\mu_{a 3}=0.015 \mathrm{~mm}^{-1}, \mu_{s 3}^{\prime}=0.8 \mathrm{~mm}^{-1}$ for the first, second, and third layers, respectively. The defect (cylin$\operatorname{der} \mathrm{B}$ ) is located in the third layer, with the center at
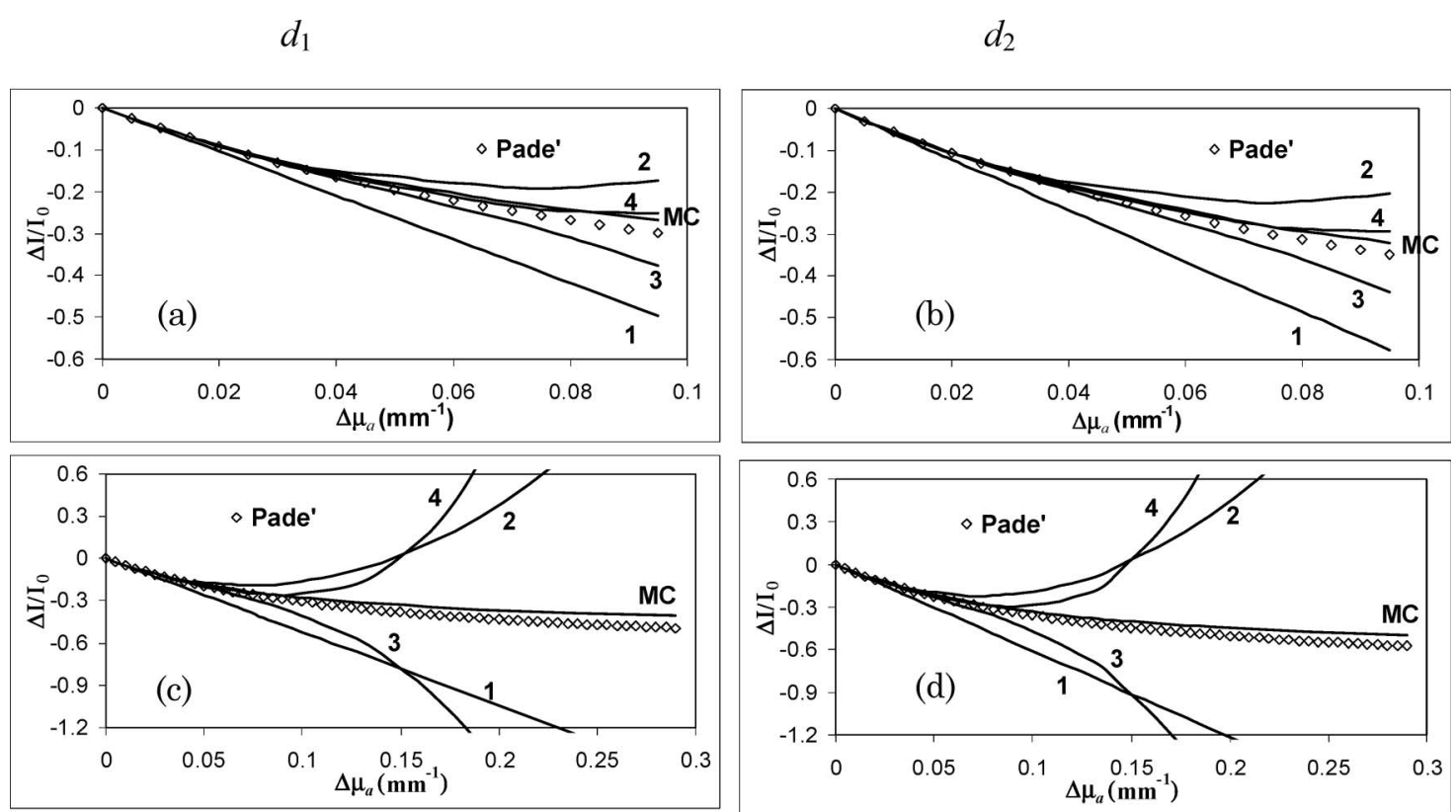

Fig. 4. As in Fig. $\underline{2}$, but the defect is cylinder B of Fig. $\underline{1}$, located in the top layer, with the center at $(x, y, z)=(7.5,8,0) \mathrm{mm}$. 

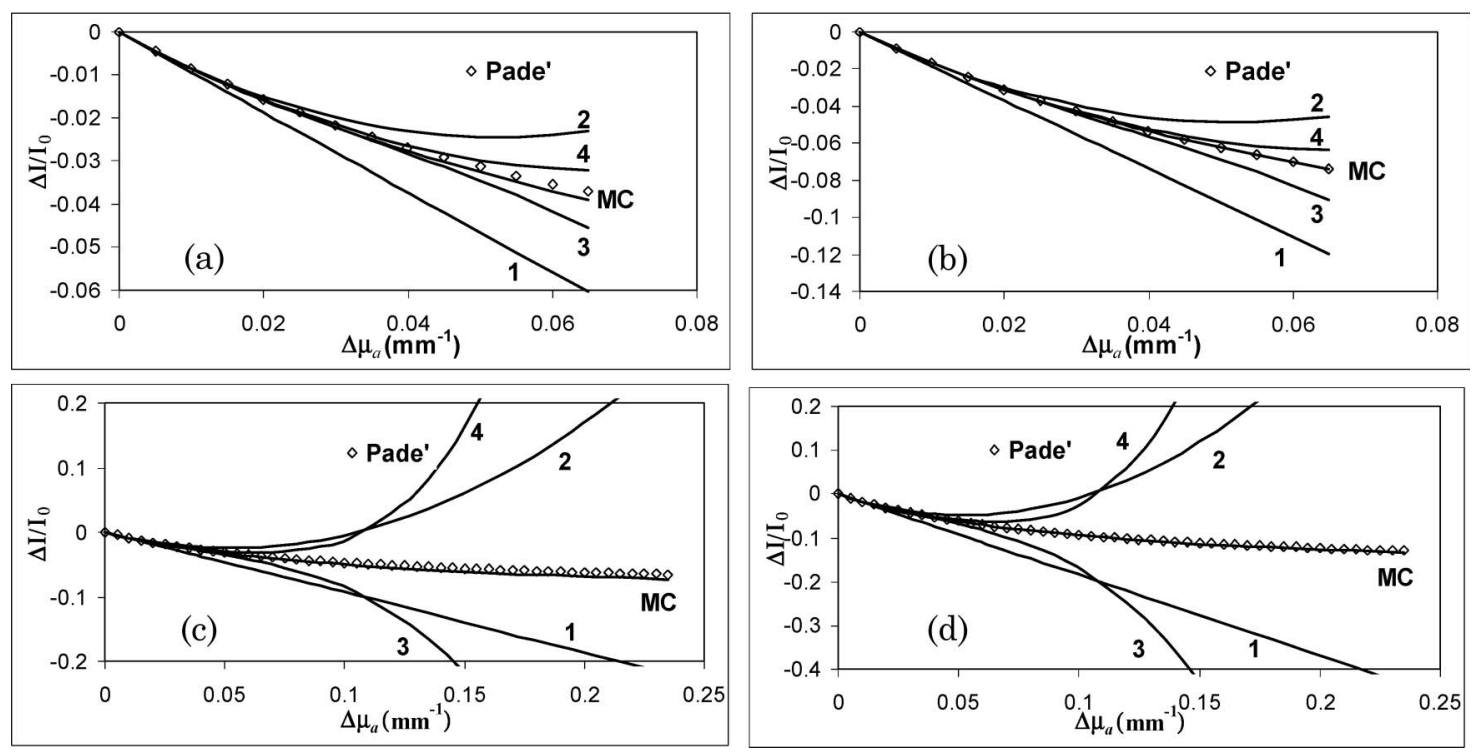

Fig. 5. As in Fig. 2 , but the defect is cylinder B of Fig. $\underline{1}$, located in the bottom layer, with the center at $(x, y, z)=(12.5,8,0) \mathrm{mm}$.

$(x, y, z)=(12,8,0) \mathrm{mm}$. Third- and fourth-order (Padé) calculations have similar agreements with MC results, which is better than 10\% (5\%) for $\Delta \mu_{a} \leq 0.06 \mathrm{~mm}^{-1}$. Padé approximants again show an outstanding agreement with MC results for larger absorption contrast also, which is better than $8 \%$ for $\Delta \mu_{a} \leq 0.2 \mathrm{~mm}^{-1}$.

In the previous results the baseline medium was a two or three-layer medium with different optical properties in each layer. We argued that the usefulness of Eq. (1) for heterogeneous media relies in its property that the effect of global and local (at the site of the defect) distribution of the optical properties are partly separated. Therefore we computed the integral of Eq. (1) by using the optical properties of the layer where the defects were embedded, since the effect of the other layer was accounted for in $\left\langle l_{i}\right\rangle$. In the previous examples we considered

\section{$d_{1}$}
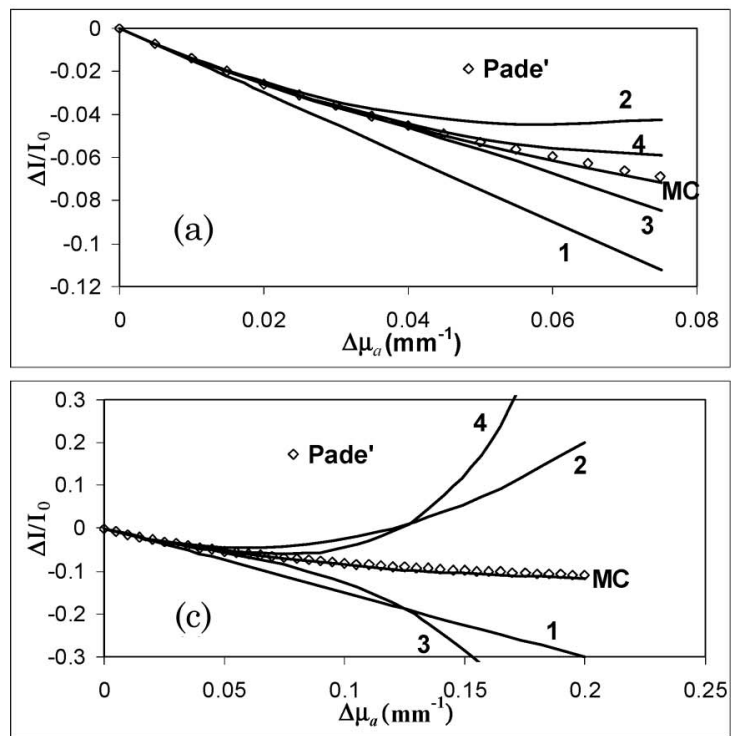

$d_{2}$
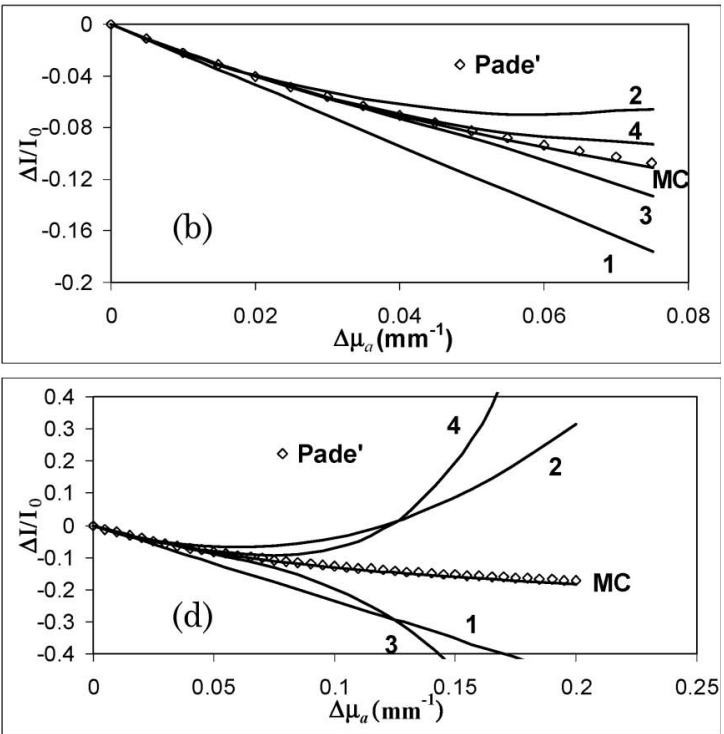

Fig. 6. Results obtained for a three-layer medium where the thicknesses of the first and second layers are 8 and $2 \mathrm{~mm}$, respectively. The optical properties are $\mu_{a 1}=0.01 \mathrm{~mm}^{-1}, \mu_{s 1}^{\prime}=0.5 \mathrm{~mm}^{-1} ; \mu_{a 2}=0.002 \mathrm{~mm}^{-1}, \mu_{s 2}^{\prime}=0.1 \mathrm{~mm}^{-1}$; and $\mu_{a 3}=0.015 \mathrm{~mm}^{-1}, \mu_{s 3}^{\prime}=0.8 \mathrm{~mm}^{-1}$ for the first, second, and third layers, respectively. The defect (cylinder B) is located in the third layer, with the center at $(x, y, z)=(12,8,0)$ mm. Changes of intensity are shown (a) at the shortest $(15 \mathrm{~mm})$ and $(\mathrm{b})$ farthest $(30 \mathrm{~mm})$ source-detector distance. In panels (c) and (d) the same curves are plotted on an expanded scale. 
locations of the defects close to the borders of different layers in order to test the validity of this hypothesis. In the following results we also tried to push the applicability of Eq. (1) a little further, and we studied several cases where the background heterogeneous medium was composed of two layers and one focal region, occupied either by cylinder $\mathrm{A}$ or $\mathrm{B}$, including a scattering perturbation. Starting from this more composite background medium we considered an absorbing perturbation in the same focal region occupied by the cylinders. If we want to apply Eq. (1), which local optical properties should we use for the calculation of the integral? In this case the local reduced scattering coefficient at the site of the cylinders is confined in relatively smaller regions than those considered for the layered media studied in the previous examples. If we use the reduced scattering coefficient of the cylinders $\left(\mu_{s_{\mathrm{cyl}}}^{\prime}\right)$ for calculation of the integral, we are assuming that light propagation occurs as if the whole layer where the cylinders are embedded had the same reduced scattering coefficient of the cylinders; while if we use the reduced scattering coefficient of the layer $\left(\mu_{s_{\text {lav }}^{\prime}}\right)$, we are disregarding the scattering change at the site of the defect. In both cases we will miscalculate the moments, but the errors must have opposite signs. Therefore we propose to carry out the calculation of $\Delta I / I_{0}$ by using both sets of values of the moments obtained with $\mu_{s_{\mathrm{cyl}}}^{\prime}$ and $\mu_{s_{\mathrm{lay}}}^{\prime}$, respectively, and afterward take the average value. This empirical rule was tested for different volumes of the defect in the range $(20,900) \mathrm{mm}^{3}$ and for different reduced scattering coefficients of the media $\left[(0.5,2) \mathrm{mm}^{-1}\right]$ and the defects $\left[\left((0.05,2.5) \mathrm{mm}^{-1}\right]\right.$. Two examples of these results are provided in Fig. 7, where the changes in $\Delta I / I_{0}$ are plotted against the absorption contrast at the source-detector distance of $30 \mathrm{~mm}$ for two different background media. The results in Fig. 7(a) refer to a two-layer medium as in Fig. 2 , which also included cylinder A located with the center at $(x, y, z)=(6.5,6.8,-4.25) \mathrm{mm}$ and having a reduced scattering coefficient of $\mu_{s_{\mathrm{cyl}}}^{\prime}=1 \mathrm{~mm}^{-1}$. Therefore this composite background medium in- cluded a positive scattering perturbation in the first layer, at the site of the cylinder. The results in Fig. $7(\mathrm{~b})$ refer to a two-layer medium having a thickness of the first layer of $10 \mathrm{~mm}$ and optical properties $\mu_{a 1}=0.01 \mathrm{~mm}^{-1}, \mu_{s 1}^{\prime}=1 \mathrm{~mm}^{-1}$, and $\mu_{a 2}^{\prime}=$ $0.015 \mathrm{~mm}^{-1}, \mu_{s 2}^{\prime}=1.5 \mathrm{~mm}^{-1}$, for the first and second layer, respectively. The background media included also cylinder $\mathrm{B}$ located with the center at $(x, y, z)=$ $(12,6.8,-4.25) \mathrm{mm}$ and having a reduced scattering coefficient of $\mu_{s_{\mathrm{cyl}}}^{\prime}=1 \mathrm{~mm}^{-1}$. Therefore this composite background medium included a negative scattering perturbation in the second layer, at the site of the cylinder. We used Eq. (1) for the calculation of the selfmoment, given the value of $\left\langle l_{i}\right\rangle$ calculated by MC code. In Fig. 7(a) the agreement of fourth-order calculations and the MC curve is better than $10 \%$ for $\Delta \mu_{a} \leq 0.07 \mathrm{~mm}^{-1}$. Padé approximants yielded excellent comparison with $\mathrm{MC}$ results with an agreement better than $1 \%$ for $\Delta \mu_{a} \leq 0.19 \mathrm{~mm}^{-1}$. In Fig. $\underline{7(\mathrm{~b})}$ fourth-order calculations matched the MC curve to within $11 \%$ for $\Delta \mu_{a} \leq 0.055 \mathrm{~mm}^{-1}$, while Padé approximants showed an agreement better than $2 \%$ for $\Delta \mu_{a} \leq 0.19 \mathrm{~mm}^{-1}$.

One important result that holds at least for values of the volumes and reduced scattering coefficients of the defects in the range $(20,900) \mathrm{mm}^{3}$ and $(0.05,2.5) \mathrm{mm}^{-1}$, respectively, is that the presence of a scattering perturbation alters the value of the mean path length $\left\langle l_{i}\right\rangle$ usually by a few percent and by no more than about $10 \%$. For example in Fig. 7(a) the mean path length within the cylinder is $\left\langle l_{i}\right\rangle=$ $2.83 \mathrm{~mm}$, which is very close to the value of mean path length calculated inside the same region when no scattering perturbation is considered in the first layer $\left(\left\langle l_{i}\right\rangle=2.82 \mathrm{~mm}\right)$. In Fig. $7(\mathrm{~b})$ the value of mean path length within the cylinder is $\left\langle l_{i}\right\rangle=2.26 \mathrm{~mm}$, and the value of mean path length within the cylinder for a homogeneous second layer is $\left\langle l_{i}\right\rangle=2.23 \mathrm{~mm}$. However, the presence of a scattering perturbation affects gradually the higher-order self-moments. For Fig. $\underline{7(\mathrm{a})}\left\langle l_{i}^{4}\right\rangle=27,310 \mathrm{~mm}^{4}$, while for the case of a homogeneous first layer $\left\langle l_{i}^{4}\right\rangle=15,340 \mathrm{~mm}^{4}$. For Fig. $\underline{7(\mathrm{~b})}\left\langle l_{i}^{4}\right\rangle=84,550 \mathrm{~mm}^{4}$, while for the case $d_{2}$

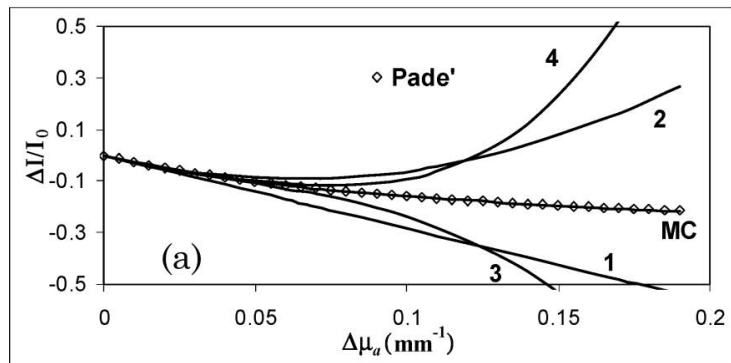

$d_{2}$

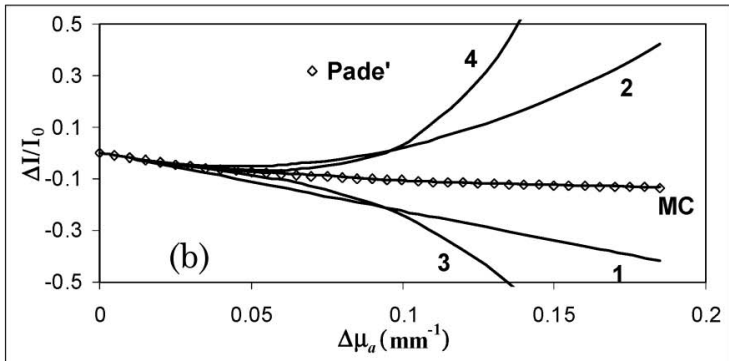

Fig. 7. In (a) we considered a background medium composed of the two-layer medium of Fig. 2 and also cylinder A (Fig. 1 ), which was located in the first layer with the center at $(x, y, z)=(6.5,6.8,-4.25) \mathrm{mm}$, and had reduced scattering coefficient of $\mu_{\mathrm{sc}}^{\prime}=1 \mathrm{~mm}^{-1}$. In (b) we considered a two-layer medium having the thickness of the first layer of $10 \mathrm{~mm}$ and optical properties $\mu_{a 1}=0.01 \mathrm{~mm}^{-1}, \mu_{s 1}^{\prime}=1 \mathrm{~mm}^{-1}$ and $\mu_{a 2}=0.015 \mathrm{~mm}^{-1}, \mu_{s 2}^{\prime}=1.5 \mathrm{~mm}^{-1}$ for the first and second layer, respectively. The background media also included also cylinder B located in the second layer with the center at $(x, y, z)=(12,6.8,-4.25) \mathrm{mm}$ and having a reduced scattering coefficient of $\mu_{\mathrm{sc}}^{\prime}=1 \mathrm{~mm}^{-1}$. The results refer to a source-detector distance $d=30 \mathrm{~mm}$. 
of a homogeneous second layer $\left\langle l_{i}^{4}\right\rangle=92,450 \mathrm{~mm}^{4}$. The reason for this behavior is found in the diffusive nature of photon migration. When the reduced scattering coefficient of a region is increased with respect to the background value, we can expect that on average the same number of detected photons will intersect the region. However, the increased value of scattering will change the distribution of path lengths $l_{i}$ of detected photons by favoring both shorter and longer path lengths. Therefore the value of the mean path length will change only to a very small extent, but the higher-order self-moments will be strongly affected by the longer path lengths. In other words, detected photons that crossed strong scattering perturbations were either reflected or trapped by the perturbation. An example of this behavior is shown in Fig. 8, where we considered a background medium and a defect as in Fig. 2 with the only difference that they were both nonabsorbing (i.e., $\mu_{a 1}=$ $\left.\mu_{a 2}=\mu_{a \mathrm{cyl}}=0\right)$. We calculated the distribution of internal path lengths $\left(l_{i}\right)$ with 10,000 detected photons when the reduced scattering coefficient of the cylinder was 0.05 and $2.5 \mathrm{~mm}^{-1}$, that is, for a negative and positive scattering perturbation, respectively. We can see that the distribution of internal path lengths for the stronger scattering cylinder is higher for very short path lengths $\left(l_{i} \leq 3 \mathrm{~mm}\right)$ and also for longer path lengths $\left(l_{i} \geq 20 \mathrm{~mm}\right)$. However, for the lower scattering cylinder there is an intermediate range of path lengths ( $\left.3 \mathrm{~mm} \leq l_{i} \leq 20 \mathrm{~mm}\right)$ with higher probability of occurrence. As a consequence we can expect little difference between the mean path lengths but a large difference between higher-order moments. In fact the first three moments are $\left\langle l_{i}\right\rangle=5.3 \mathrm{~mm}$, $\left\langle l_{i}^{2}\right\rangle=69 \mathrm{~mm}^{2},\left\langle l_{i}^{3}\right\rangle=1290 \mathrm{~mm}^{3}$ for the lower scattering cylinder, and $\left\langle l_{i}\right\rangle=5.7 \mathrm{~mm},\left\langle l_{i}^{2}\right\rangle=152 \mathrm{~mm}^{2}$, $\left\langle l_{i}^{3}\right\rangle=6320 \mathrm{~mm}^{3}$ for the higher scattering cylinder.

The property that a scattering perturbation will change $\left\langle l_{i}\right\rangle$ only to small extent $(\leq 10 \%)$ can be used to study the effect of a colocalized scattering and absorbing perturbation, following the empirical rule used in Fig. 7, with the value of mean path length calculated inside the defect when there is no scattering contrast. Note that by this method we can study a colocalized scattering and absorbing perturbation,

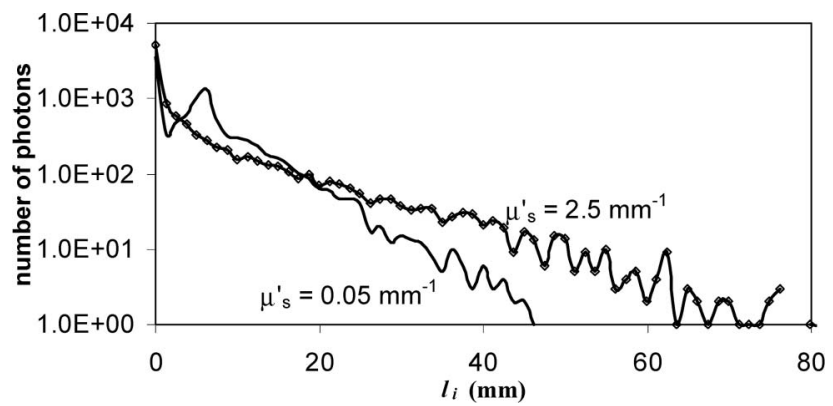

Fig. 8. Distribution of internal path lengths calculated with 10,000 detected photons for a background medium and a defect as in Fig. $\underline{2}$, but both nonabsorbing. The distributions refer to two values of the reduced scattering coefficient of the cylindrical defect, as indicated. but nothing can be said about a pure scattering perturbation.

Finally, we emphasize the robustness of the path length approach for studying higher-order perturbation theory by showing one result in the slab geometry, which is used by several groups in breast imaging $[25,32,33]$. In Fig. 9 is shown a schematic of a heterogeneous slab having thickness of $40 \mathrm{~mm}$ and optical properties $\mu_{a}=0.005 \mathrm{~mm}^{-1}$ and $\mu_{s}^{\prime}=0.5 \mathrm{~mm}^{-1}$. A focal spherical region $(10 \mathrm{~mm}$ in diameter) located with the center at $(x, y, z)=(12,7,0) \mathrm{mm}$ and having $\mu_{a 1}=0.1 \mathrm{~mm}^{-1}$ was included in the background medium. This focal region with very high absorption coefficient may simulate the presence of a network of blood vessels. Another spherical region $(10 \mathrm{~mm}$ in diameter) located at $(x, y, z)=(20,0,0) \mathrm{mm}$ was considered absorbing perturbation. Three detectors were placed in transmittance at the distances from the $x$ axis of 0,10 , and $20 \mathrm{~mm}$. In Fig. $10 \Delta I / I_{0}$ is plotted against the absorption contrast $\Delta \overline{\mu_{a}}$ between the central spherical region and background for the detector $d_{1}$ and $d_{3}$ (Fig. 9), respectively. The agreement of the fourth-order calculation with $\mathrm{MC}$ results was better than $12 \%$ for $\Delta \mu_{a}<0.05 \mathrm{~mm}^{-1}$, while Padé approximants showed an agreement with the MC curve of better than $1 \%$ for $\Delta \mu_{a}<0.2 \mathrm{~mm}^{-1}$. These examples in layered and slab geometries strongly suggest that similar results can be found in other geometries used in NIRS and DOT.

\section{Discussion and Conclusions}

In this work we have applied Eq. (1), proposed in our previous work [22], for studying perturbation theory in layered and slab diffusive media. Although Eq. (1) was proposed as a heuristic formula for the calculation of higher-order self-moments of focal inclusions

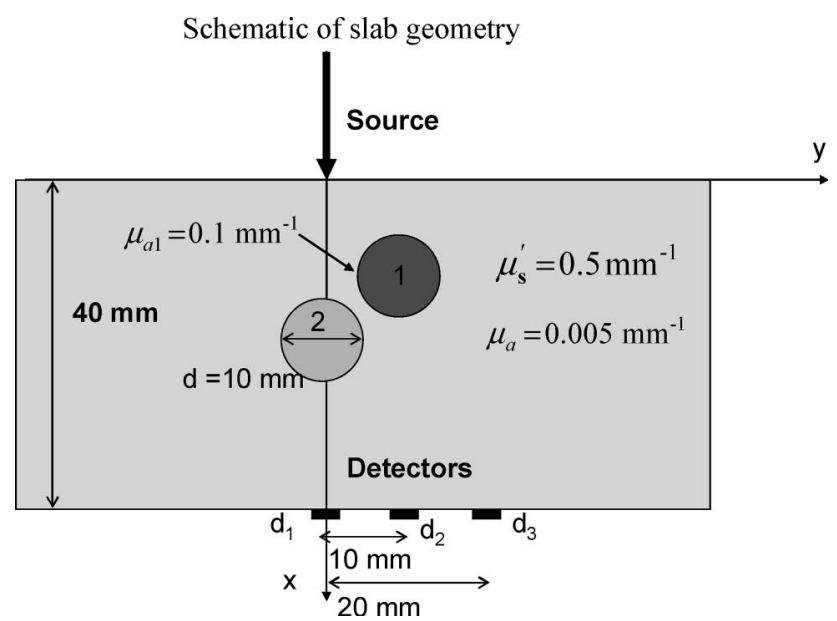

Fig. 9. Schematic of the heterogeneous slab geometry composed by a slab with $\mu_{a}=0.005 \mathrm{~mm}^{-1}, \mu_{s}^{\prime}=0.5 \mathrm{~mm}^{-1}$, and a spherical region (1) with $\mu_{a 1}=0.1 \mathrm{~mm}^{-1}$ and the same reduced scattering coefficient. The spherical region is located with the center at $(x, y, z)=(12,7,0) \mathrm{mm}$. Another spherical region (2), located with the center at $(x, y, z)=(20,0,0) \mathrm{mm}$ was considered an absorbing perturbation. Both spherical regions have the same diameter of $10 \mathrm{~mm}$. The thickness of the slab is $40 \mathrm{~mm}$. Three detectors are placed in transmittance as indicated in the figure. 

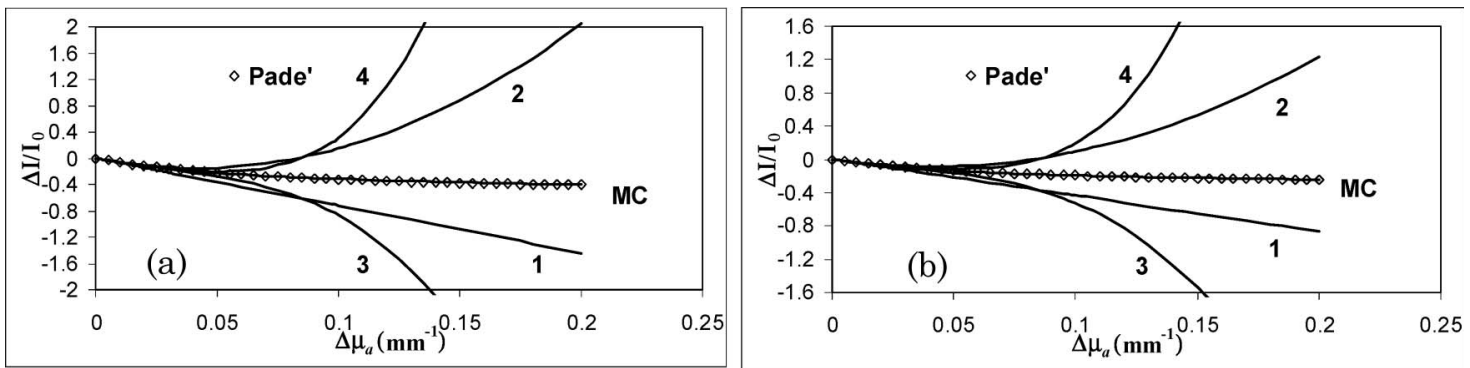

Fig. 10. For the slab geometry the changes of intensities $\Delta I / I_{0}$ are plotted at the detector (a) $d_{1}$ and (b) $d_{3}$ (Fig. $\underline{9}$ ).

in homogeneous media, in this work we have shown its potential to describe photon migration in heterogeneous media. In particular we have considered the layered and the slab geometries, since they are typically used in brain and breast imaging, respectively. By considering different combinations of the optical and geometrical properties of the media and the inclusions, we have found that Eq. (1) can be effectively used to extend the limitations of first-order theory. There is always a range of contrasts $d \Delta \mu_{a}$, where $d$ is an effective diameter of the inclusion and $\Delta \mu_{a}$ the absorption contrast between inclusion and local background, where fourth-order perturbation theory offers a clear improvement with respect to first-order theory. We have found that in the range of contrasts up to $d \Delta \mu_{a}<0.4-0.8$, fourth-order calculations yields results accurate to within about $10 \%$. It is also rather straightforward to understand when fourthorder calculations are affected by larger errors. In fact, for all the trends of $\Delta I / I_{0}$ as the function of $\Delta \mu_{a}$ presented in this work, the value of $\Delta \mu_{a}$ where the first derivative is zero is usually affected by an error of $10 \%-20 \%$. Therefore, to have accurate fourth-order calculations, the values of $\Delta \mu_{a}$ are those in a range where

$$
\frac{\partial\left(\Delta I / I_{0}\right)}{\partial\left(\Delta \mu_{a}\right)} \leq 0
$$

We have also used the method of Padé approximants, and we have found that it always shows the best overall agreement with MC results. In the range of absorption contrasts where fourth-order theory yields useful results (accurate to within 10\%), the method of Padé approximants also shows a similar accuracy. The clear advantage of Padé approximants is for larger absorption contrasts, in the range $(0.1,0.2-0.3) \mathrm{mm}^{-1}$. However, a problem for the application of this method is caused by the occurrence of zeros in the denominator of Eq. (3) for values of $\Delta \mu_{a}$ in the range of interest. Zeros of the rational function in Eq. (3) are sensitive to the coefficient of MacLaurin expansion [Eq. (2)]. In particular, for the values of $c_{n}$ used in this work, no zero was ever found in all the cases presented. When we changed the values of the scaling parameters to $c_{1}=1.5$, $c_{2}=3.1$, and $c_{3}=10$, we systematically found one zero located around $\Delta \mu_{a} \approx 0.1 \mathrm{~mm}^{-1}$. Even though this absorption contrast is rather strong and is not usually found in DOT, we cannot exclude in general that a zero cannot be found in a lower range of $\Delta \mu_{a}$. The presence of zeros should be addressed before incorporating Padé approximants as a forward model in a code for optical properties reconstruction. A zero of Eq. (3) translates into the presence of a vertical asymptote in the plot of $\Delta I / I_{0}$ against $\Delta \mu_{a}$. However, we note that even if a vertical asymptote is present the incorrect values of $\Delta I / I_{0}$ around the zero can be easily spotted. In fact, on both sides of the asymptote the decreasing trend of $\Delta I / I_{0}$ against $\Delta \mu_{a}$ is abruptly interrupted and reversed. Those values of $\Delta I / I_{0}$ characterized by a reversed trend usually cover a range of $\Delta \mu_{a}$ surrounding the zero with a width of $\sim 0.01-$ $0.02 \mathrm{~mm}^{-1}$. Once spotted, the wrong points can be eliminated by using an interpolating function. At this level of the investigation this can be a safe way to apply the method of Padé approximants as a forward model solver.

Finally we note that in this work we have reduced the problem of $n$ th-order perturbation theory to the estimation of the mean path length $\left\langle l_{i}\right\rangle$; therefore the computational complexity of the proposed theory is the same as first-order perturbation theory (Born approximation). Analytical solutions for $\left\langle l_{i}\right\rangle$ are available only for regular geometries, and numerical methods are required for more complex geometries. Therefore it may be possible that the proposed method can be used in conjunction with some numerical methods to estimate the effect of local inclusions in arbitrary geometries, beyond the limits of firstorder perturbation theory. We have also tried to extend the range of applicability of Eq. (1) for colocalized scattering and absorbing perturbations, and we found very encouraging results in the layered geometry. Last, we want to mention that the proposed method is suitable for dealing with multiple defects, as we showed in our previous work [23].

\section{Appendix A}

The perturbative $\mathrm{DE}$ for absorbing inclusions is formally equivalent to the $\mathrm{DE}$, and it is written as

$$
-\nabla \cdot\{D(\mathbf{r}) \nabla[\Delta \phi(\mathbf{r})]\}+\mu_{a}(\mathbf{r}) \Delta \phi(\mathbf{r})=-\Delta \mu_{a}(\mathbf{r}) \phi_{f i}(\mathbf{r}),
$$


where $D(\mathbf{r})$ is the diffusion coefficient; $\mu_{a}(\mathbf{r})$ and $\Delta \mu_{a}(\mathbf{r})$ are the absorption coefficient and the absorption perturbation, respectively; $\Delta \phi(\mathbf{r})=\phi_{f i}(\mathbf{r})-$ $\phi_{i n}(\mathbf{r})$, where $\phi_{i n}(\mathbf{r})$ is the Green's function of the fluence rate for the baseline (initial state) medium and $\phi_{f i}(\mathbf{r})$ is the Green's function of the fluence rate after the absorption perturbation has occurred (final state). The fluence rate is normalized to the source strength; therefore $[\phi]=L^{-2}$. The solution of Eq. (A1) is expressed in integral form by the convolution theorem:

$$
\phi_{f i}(\mathbf{r})=\phi_{i n}(\mathbf{r})-\int_{V} \Delta \mu_{a}\left(\mathbf{r}_{1}\right) \phi_{i n}\left(\mathbf{r}, \mathbf{r}_{1}\right) \phi_{f i}\left(\mathbf{r}_{1}\right) \mathrm{d} \mathbf{r}_{1},
$$

where $V$ is the volume where the absorption perturbation is located. Equation (A2) is a Fredholm equation of the second kind, and its solution is provided by the Neumann series. The terms of the Neumann series can be rearranged to derive the moments of the generalized temporal point spread function. The general expression of the self-moments for small absorbing inclusions (i.e., inclusions having sizes much smaller than the distance between inclusion and both source and detector) is given by

$$
\begin{aligned}
\left\langle l_{i}^{n}\right\rangle \approx & \frac{n !\left\langle l_{i}\right\rangle}{V_{i}} \int_{V_{i}} d \mathbf{r}_{1} \int_{V_{i}} \phi_{0}\left(\mathbf{r}_{1}, \mathbf{r}_{2}\right) d \mathbf{r}_{2} \int_{V_{i}} \phi_{0}\left(\mathbf{r}_{2}, r_{3}\right) d \mathbf{r}_{3} \\
& \ldots \int_{V_{i}} \phi_{0}\left(\mathbf{r}_{n-1}, \mathbf{r}_{n}\right) d \mathbf{r}_{n},
\end{aligned}
$$

where $V_{i}$ and $\left\langle l_{i}\right\rangle$ are the volume and the mean path length inside the inclusion, respectively. Since $\phi_{0}\left(\mathbf{r}_{1}, \mathbf{r}_{2}\right)$ is the fluence rate when the source and the field points are inside the same region (therefore indefinitely close), we should question whether the solution of the DE can be used as the integrand and, if not, whether Eq. (A3) is a valid expression of the moments within the RTE. It is possible to prove that Eq. (A3) yields the correct values of the self-moments up to $n=2$; however the Green's function of the RTE for the fluence rate should be used for calculation of the multiple integral. For higher perturbation orders, the moments derived within the RTE, which are not formally equivalent to Eq. (3), should be used, following an analogous procedure [22]. Since the correct expressions of the moments for $n>2$ derived within the RTE are not useful for practical calculations, we proposed the heuristic formula given in Eq. (1). Finally, we note that the perturbative RTE can also be solved by using the formalism of the transition operator or $\mathrm{T}$ matrix as shown by other investigators [34].

This research is supported by National Science Foundation (NSF) award IIS-0713506, NIH R21DA021817, and NIH CA95885. F. Martelli acknowledges funding from the European Community's Se- venth Framework Program [FP7/2007-2013] under grant agreement HEALTH-F5-2008-201076.

\section{References}

1. S. R. Arridge, "Optical tomography in medical imaging," Inverse Probl. 15, R41-R93 (1999).

2. S. R. Arridge, M. Cope, and D. T. Delpy, "The theoretical basis for the determination of optical pathlength in tissue: temporal and frequency analysis," Phys. Med. Biol. 37, 1531-1560 (1992).

3. D. A. Boas, M. A. O'Leary, B. Chance, and A. G. Yodh, "Scattering of diffuse photon density waves by spherical inhomogeneities within turbid media: analytic solution and applications," Proc. Natl. Acad. Sci. USA 91, 4887-4891 (1994).

4. D. A. Boas, M. A. O'Leary, B. Chance, and A. G. Yodh, "Detection and characterization of optical inhomogeneities with diffuse photon density waves: a signal to noise analysis," Appl. Opt. 36, 75-92 (1997)

5. P. N. den Outer, Th. M. Nieuwenhuizen, and A. Lagendijk, "Location of objects in multiple-scattering media," J. Opt. Soc. Am. A 10, 1209-1218 (1993).

6. S. A. Walker, D. A. Boas, and E. Gratton, "Photon density waves scattered from cylindrical inhomogeneities: theory and experiments," Appl. Opt. 37, 1935-1944 (1998).

7. A. Kienle, T. Glanzmann, G. Wagnieres, and H. V. Bergh, "Investigation of two-layered turbid medium with time-resolved reflectance," Appl. Opt. 37, 6852-6862 (1998).

8. F. Martelli, A. Sassaroli, S. Del Bianco, Y. Yamada, and G. Zaccanti, "Solution of the time-dependent diffusion equation for layered random media by the eigenfunction method," Phys. Rev. E 67, 056623 (2003).

9. J. Sikora, A. Zacharopoulos, A. Douiri, M. Schweiger, L. Horesh, A. R. Arridge, and J. Ripoll, "Diffuse photon propagation in multilayered geometries," Phys. Med. Biol. 51, 497-516 (2006).

10. J. Ripoll, V. Ntziachristos, J. P. Culver, D. N. Pattanayak, A. G. Yodh, and M. Nieto-Vesperinas, "Recovery of optical parameters in multiple-layered diffusive media: theory and experiments," J. Opt. Soc. Am. A 18, 821-830 (2001).

11. K. Ren, G. S. Abdoulaev, G. Bal, and A. H. Hielscher, "Algorithm for solving the equation of radiative transfer in the frequency domain," Opt. Lett. 29, 578-580 (2004).

12. S. R. Arridge, M. Schweiger, M. Hiraoka, and D. T. Delpy, "A finite element approach to modelling photon transport in tissue," Med. Phys. 20, 299-309 (1993).

13. H. Jiang, K. D. Paulsen, U. L. Osterberg, B. W. Pogue, and M. S. Patterson, "Optical image reconstruction using frequency-domain data: simulations and experiments," J. Opt. Soc. Am. 13, 253-266 (1996).

14. R. L. Barbour, H. L. Graber, Y. Pei, S. Zhong, and C. H. Schmitz, "Optical tomographic imaging of dynamic features of dense-scattering media," J. Opt. Soc. Am. 18, 3018-3036 (2001).

15. Y. Yao, Y. wang, Y. Pei, W. Zhu, and R. L. Barbour, "Frequency domain optical imaging of absorption and scattering distributions by a Born iterative method," J. Opt. Soc. Am. A 14, 325-342 (1997)

16. D. A. Boas, J. P. Culver, J. J. Stott, and A. K. Dunn, “Three dimensional Monte Carlo code for photon migration through complex heterogeneous media including the adult human head," Opt. Express 10, 159-170 (2002).

17. L. Wang, S. L. Jacques, and L. Zheng, "MCML-Monte Carlo modeling of light transport in multi-layered tissues," Comput. Methods Programs Biomed. 47, 131-146 (1995).

18. R. Graaf, M. H. Koelink, F. F. M. de Mul, W. G. Zijlstra, A. C. M. Dassel, and J. G. Aarnoudse, "Condensed Monte Carlo 
simulations for the description of light transport," Appl. Opt. 32, 426-434 (1993).

19. G. Zaccanti, "Monte Carlo study of light propagation in optically thick media: point source case," Appl. Opt. 30, 2031-2041 (1991).

20. M. R. Ostermeyer and S. L. Jacques, "Perturbation theory for diffuse light transport in complex biological tissues," J. Opt. Soc. Am. A 14, 255-261 (1997).

21. D. A. Boas, "A fundamental limitation of linearized algorithms for diffuse optical tomography," Opt. Express 1, 404-413 (1997).

22. A. Sassaroli, F. Martelli, and S. Fantini, "Perturbation theory for the diffusion equation by use of the moments of the generalized temporal point-spread function: I. Theory," J. Opt. Soc. Am. A 23, 2105-2118 (2006).

23. A. Sassaroli, F. Martelli, and S. Fantini, "Perturbation theory for the diffusion equation by use of the moments of the generalized temporal point-spread function: II. Continuous wave results," J. Opt. Soc. Am. A 23, 2119-2131 (2006).

24. B. Wassermann, "Limits of high-order perturbation in timedomain optical mammography," Phys. Rev. E 74, 031908 (2006).

25. D. Grosenick, A. Kummrow, R. Macdonald, P. M. Schlag, and H. Rinneberg, "Evaluation of higher-order time domain perturbation theory of photon diffusion on breast-equivalent phantoms and optical mammograms," Phys. Rev. E 76, 061908 (2007).

26. V. Chernomordik, D. Hattery, A. Gandjbakhche, A. Pifferi, P. Taroni, A. Torricelli, G. Valentini, and R. Cubeddu, "Quantification by random walk of the optical parameters of nonlo- calized abnormalities embedded within tissuelike phantoms," Opt. Lett. 25, 951-953 (2000).

27. M. Xu, W. Cai, and R. R. Alfano, "Multiple passages of light through an absorption inhomogeneity in optical imaging of turbid media," Opt. Lett. 29, 1757-1759 (2004).

28. L. Spinelli, A. Torricelli, A. Pifferi, P. Taroni, and R. Cubeddu, "Experimental tests of a perturbation model for timeresolved imaging of diffusive media," Appl. Opt. 42, 31453153 (2003).

29. W. H. Press, S. A. Teukolsky, W. T. Vetterling, and B. P. Flannery, Numerical Recipes, 2nd ed. (Cambridge U. Press, 1992).

30. F. Martelli, S. Del Bianco, and G. Zaccanti, "Perturbation model of light propagation through diffusive layered media," Phys. Med. Biol. 50, 2159-2166 (2005).

31. S. Carraresi, T. S. M. Shatir, F. Martelli, and G. Zaccanti, "Accuracy of a perturbation model to predict the effect of scattering and absorbing inhomogeneities on photon migration," Appl. Opt. 40, 4622-4632 (2001).

32. S. Fantini, E. L. Heffer, V. E. Pera, A. Sassaroli, and N. Liu, "Spatial and spectral information in optical mammography," Technol. Cancer Res. Treat. 4, 471-482 (2005).

33. P. Taroni, D. Comelli, A. Pifferi, A. Torricelli, and R. Cubeddu, "Absorption of collagen: effects on the estimate of breast composition and related diagnostic implications," J Biomed. Opt. 12, 014021 (2007).

34. A. D. Kim and J. C. Schotland, "Self-consistent scattering theory for the radiative transport equation," J. Opt. Soc. Am. A 23, 596-602 (2006). 